\title{
From Biomimetic Mineralization to Carbonization: Fabricating Heterostructured Porous Carbon Materials with MOF Encapsulated Bacteria
}

\author{
Shaobo Li, ${ }^{1}$ Xiaoshuang Zhou, ${ }^{4}$ Zhuo Chen, ${ }^{1}$ Rangana Jayawickramage, ${ }^{1}$ Sampath B. Alahakoon, ${ }^{1}$ Xin \\ Meng, ${ }^{2}$ John Ferraris, ${ }^{1}$ Ronald A. Smaldone, ${ }^{1}$ Jeremiah J. Gassensmith*1,3 \\ ${ }^{1}$ Department of Chemistry and Biochemistry, ${ }^{2}$ Department of Electrical Engineering, ${ }^{3}$ Department of Biomedical Engineer- \\ ing, The University of Texas at Dallas, 800 W. Campbell Road, Richardson, Texas 75080, United States. ${ }^{4}$ Jiangsu Collabora- \\ tive Innovation Center of Photovoltaic Science and Engineering, Changzhou University, Changzhou 213164, China
}

\section{Supporting Information Placeholder}

\section{Introduction}

Hierarchical porous carbons (HPC) are a class of materials that are fabricated to reach high surface area, multi-scale porosity, heteroatom doping, excellent conductivity, and outstanding stability - characteristics that are highly sought after in adsorbents, catalysis, energy conversion and energy storage. ${ }^{1-7}$ Conventional fabrications of HPCs rely on utilizing synthetic inorganic/organic templates or chemical activation that generally require multiple steps beginning with template syntheses, followed by precursor infiltration, carbonization, and template removal to generate the desired heterogenous porosity. ${ }^{1-3,8}$ These tedious synthetic processes inevitably increase the complexity and cost of HPC preparation thus hindering the eventual mass production and commercialization of such promising materials. Over the previous two decades, metal organic frameworks (MOFs) have become a popular architecture for advanced materials owing to their highly customizable pore structure, composition, and straight forward synthetic strategies. ${ }^{9-11}$ MOFs are also recognized as promising precursors for porous carbons. Because of their characteristic

porous nature as well as high carbon content, MOFs enable one-step conversion to porous carbons possessing high surface area with an inner-connected porous architecture..$^{12-17}$

Scheme 1. Schematic illustration of various bacteria shapes and assemblies.

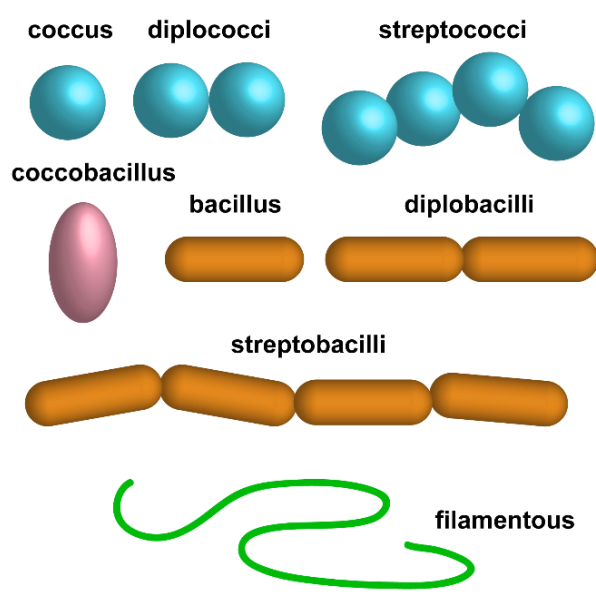

In recent years, templated MOF synthesis with biologically derived substrates has begun producing an ever-growing array of bio-MOF composites featuring morphology and size that are dictated, in part, by the underlying biological material used. In addition to potential benefits such as environmental friendliness, low cost, and high efficiency, ${ }^{18-27}$ the available biomass to template MOF syntheses is extraordinarilyviruses $^{22-23,28}$ and yeast ${ }^{20-21,27}$ have already been shown to be outstanding templates to produce core-shell systems - and researchers have only begun to explore the potential uses of biomass as homogenous and low-cost templates for new MOF architectures. Bacteria's high reproduction rate and natural abundance, make it an ideal source of cheap precursor materials - indeed our body contains at least three times more bacterial cells than human cells! In addition to its ubiquity, the diversity of shapes and assemblies (Scheme 1) can provide multiple morphological templates for biomimetic MOF syntheses. 
Scheme 2. Schematic illustration of synthetic processes of CEZ8 HPC (top) and CZ8 HPC (bottom), which used E.coli@ZIF-8 composite and pristine ZIF-8 as precursors, respectively.

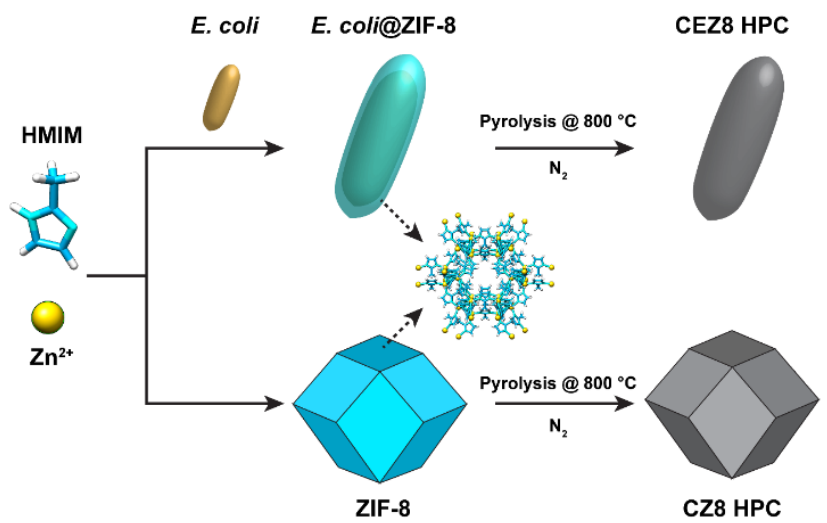

Herein, we report a bio-inspired preparation of MOF-derived HPCs driven by a robust bacterial template. A non-pathogenic non-toxic laboratory strain of Escherichia coli was employed to construct a thin crystalline shell of zeolitic imidazolate framework-8 (ZIF-8), a widely used MOF carbonization precursor owing to its extraordinary high surface area and stability. ${ }^{11}$ Interestingly, the resultantE.coli@ZIF-8 (EZ8) composite possess not only well-defined micropores, but also mesopores and macropores that can be attributed to the disturbed crystal growth on the cell membrane. As anticipated, this pore hierarchy can be retained after carbonization of EZ8. The asobtained carbonized EZ8 (CEZ8) is considerably more mesoporous compared to its parent material. The electrochemical characteristics of CEZ8 were tested in a standard threeelectrode system, and we observed superior specific capacity compared to carbonized pristine ZIF-8 (denoted as CZ8).

\section{Results \& Discussion}

\section{Preparation of E.coli@ZIF-8 (EZ8)}

The CEZ8 HPC was produced from core-shell structured EZ8 composite precursor, which was fabricated using direct biomimetic template methods that we and others have developed. This new approach differs from previous efforts to grow ZIF-8 on bacteria ${ }^{27}$ in a number of important ways. In particular, the synthesis yields E.coli@ZIF-8 with a more homogeneous surface and thinner ZIF-8 shell (Figure 1a and c). Furthermore, our one-pot synthesis procedure is fast (30 minutes) and works at ambient conditions in a purely aqueous system without methanolic solvent, which can cause cell lysis and the breakdown of the bacteria. In a typical synthesis, purified E. coli (1 $\mathrm{mg}$ ) was mixed with an aliquot of 2-methylimidazole (HMIM) aqueous solution $(1600 \mathrm{mM})$ followed by an aliquot of zinc acetate aqueous solution $(20 \mathrm{mM})$. The mixture was shaken for $20 \mathrm{~s}$ and left on the benchtop for $30 \mathrm{~min}$. The as-formed EZ8 was collected by centrifugation at $4300 \times \mathrm{g}$ then washed twice with ultrapure water. As shown in Figure 1, EZ8 retains the rod-like morphology of bacteria (Figure 1a), and the light/dark contrast in TEM micrograph clearly illustrates the core-shell structure (Figure 1c). High magnification TEM micrograph (Figure S1a) illustrates clearly that the shells are composed of closely packed nanocrystals. To analyze crystallinity and phase purity, EZ8 dry powder was analyzed by powder X-ray diffraction (PXRD) (Figure 2a), and the result shows the pure sodalite crystalline phase of ZIF-8.

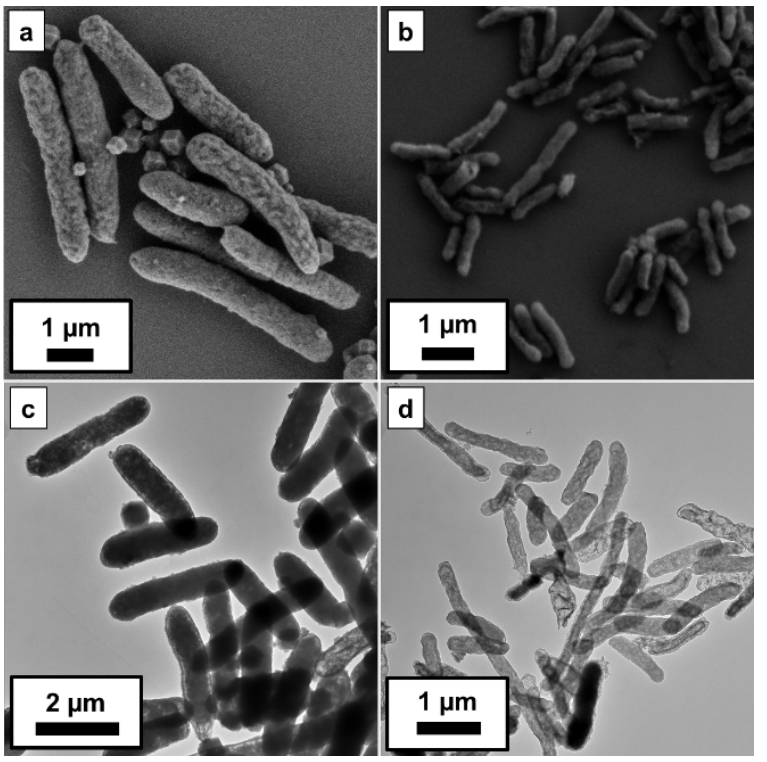

Figure 1. SEM micrographs of a) uncarbonized EZ8 and b) the carbonized CEZ8; TEM micrographs of c) EZ8 and d) CEZ8.

A nitrogen sorption isotherm was collected at $77 \mathrm{~K}$ to obtain the specific surface area and pore size distribution of EZ8 and pristine ZIF-8, as shown in Figure S3. The as-prepared pristine ZIF-8 possesses a typical type I isotherm with a sharp uptake at low relative pressure $(<0.03)$, indicating the presence of micropores (Figure S3a); however, in addition to the similar microporous pattern, EZ8 also contains a H3 hysteresis loop at relative pressure of 0.45 to 1.0 , which typically indicates capillary condensation of adsorbate $\left(\mathrm{N}_{2}\right)$ in mesopores. The calculated BET surface area of ZIF-8 and EZ-8 are 1842 and 1209 $\mathrm{m}^{2} / \mathrm{g}$, respectively. As shown in Figure $\mathrm{S} 3 \mathrm{~b}$, the pore distribution analysis uncovered two intense peaks at $10.8 \AA$ in both ZIF-8 and EZ8, while a series of lower peaks in the mesopore range $(2-50 \mathrm{~nm})$ indicate hierarchical pore characteristics in EZ8. We attributed the presence of mesopores to crystalline defects arising from the drastic crystallization on the surface of bacterial cell membranes, and we anticipated that the mesoporous characteristics of the shell can be retained after carbonization.
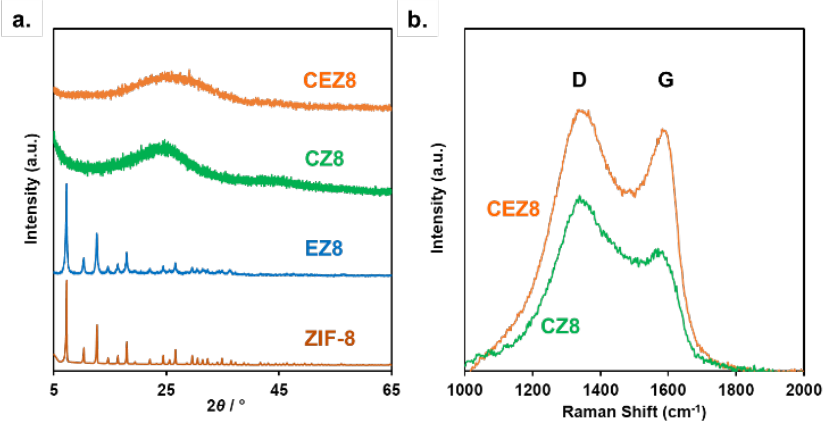

Figure 2. a) Powder X-ray diffraction patterns of EZ8 and CEZ8; b) Raman spectra of CZ8 and CEZ8. 

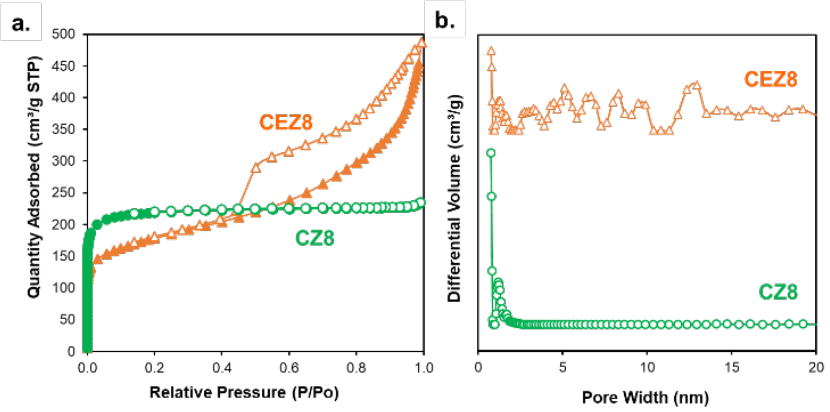

Figure 3. a) $\mathrm{N}_{2}$ sorption analysis of CZ8 and CEZ8; b) porosity distribution of CZ8 and CEZ8.

\section{Preparation of carbonized E.coli@ZIF-8 (CEZ8)}

The carbonization was carried out with by temperatureprogramed pyrolysis for both EZ8 and ZIF-8. Materials were dried at $120{ }^{\circ} \mathrm{C}$ under dynamic vacuum overnight prior to pyrolysis in a tube furnace with a nitrogen atmosphere. The temperature was raised with a $5{ }^{\circ} \mathrm{C} / \mathrm{min}$ ramp to $350{ }^{\circ} \mathrm{C}$ and kept at $350{ }^{\circ} \mathrm{C}$ for $2 \mathrm{~h}$. We utilized this process to ablate bacterial matter without impacting the stability of the ZIF-8 shells, owing to the large thermal stability differences between $E$. coli and ZIF-8 as revealed by TGA (Figure S4). Following bacterial template ablation, the furnace was thermally ramped to 800 ${ }^{\circ} \mathrm{C}$ with the same rate and kept at $800{ }^{\circ} \mathrm{C}$ for $4 \mathrm{~h}$. The carbonized products appeared as black powder and were washed with $2 \mathrm{M} \mathrm{HCl}$ and $10 \% \mathrm{HF}$, respectively, to remove residual zinc in the products. The carbonizedE.coli@ZIF-8, denoted as CEZ8, retained rod-like morphology of EZ8, though a certain level of shrinkage (Figure 1b) was observed. TEM (Figure 1d) revealed an enhanced transparency at the inner part of CEZ8 rods. The hollowness of these rod-like structures indicates the ablation of the bacterial template. At high magnification in the TEM (Figure S1b), we observed a large number of high contrast dots on the surface of the CEZ8 rods, which can be attributed to defects or nanopores that were generated during carbonization. These observations are consistent with the hysteresis loop in the $\mathrm{N}_{2}$ sorption isotherm (Figure $3 \mathrm{a}$ ) and pore analysis that shows peaks in the mesoporous range (Figure 3b). The microstructure of carbonized materials was characterized by PXRD (Figure 2a). Both CEZ8 and CZ8 only contain a broad peak at about $25^{\circ}$, which can be attributed to the (002) peak of graphitic carbon. The content of graphitic carbon in comparison to amorphous carbon was revealed by Raman spectroscopy (Figure $2 \mathrm{~b}$ ), and the $\mathrm{I}_{\mathrm{D}} / \mathrm{I}_{\mathrm{G}}$ values were calculated as 1.07 for CEZ8 and 1.45 for CZ8, indicating that E.coli@ZIF-8 composite can produce higher content of graphitic carbon than pristine ZIF-8.

\section{Electrochemical Characterization}

Owing to its high content of graphitic carbon and welldeveloped hierarchical porosity, we were encouraged to investigate the electrochemical properties of CEZ8 in energy storage. Therefore, a standard three electrode cell with $\mathrm{Ag} / \mathrm{AgCl}$ and platinum mesh as reference electrode and counter electrode, $6 \mathrm{M} \mathrm{KOH}$ aqueous solution as electrolyte, was carried out to evaluate the capacitive behavior of CEZ8, and CZ8 as comparison. Cyclic voltammograms (Figure $4 \mathrm{a}$ and b) were recorded with the voltage window from -0.6 to $0.2 \mathrm{~V}$, and specific capacitance was calculated accordingly, as illustrated in Figure 4c and Table S1. We found that CZ8 contained the typical rectangular $\mathrm{CV}$ of electrostatic double-layer capacitors whereas the CV of CEZ8 appears to show pseudocapacitance from the carbonized bacteria substrate. ${ }^{29}$ The highest specific capacitance of CEZ8 and CZ8 were obtained as 163 and 87 $\mathrm{F} / \mathrm{g}$, at scan rate of $5 \mathrm{mV} / \mathrm{s}$, indicating a superior capacitive behavior of CEZ8. Though CEZ8 contains a lower specific surface area comparing to CZ8 $\left(645 \mathrm{~m}^{2} / \mathrm{g}\right.$ vs. $\left.841 \mathrm{~m}^{2} / \mathrm{g}\right)$, the superior performance is attributed to its hierarchical porosity, in particular the high content of mesopores as well as its higher content graphitic carbon, which greatly improves the ion transfer efficiency. Moreover, both CEZ8 and CZ8 show excellent cyclability as they demonstrate $94.5 \%$ and $95 \%$ retention after 2000 cycles (Figure 4d), which we ascribe to their robust structural stability.
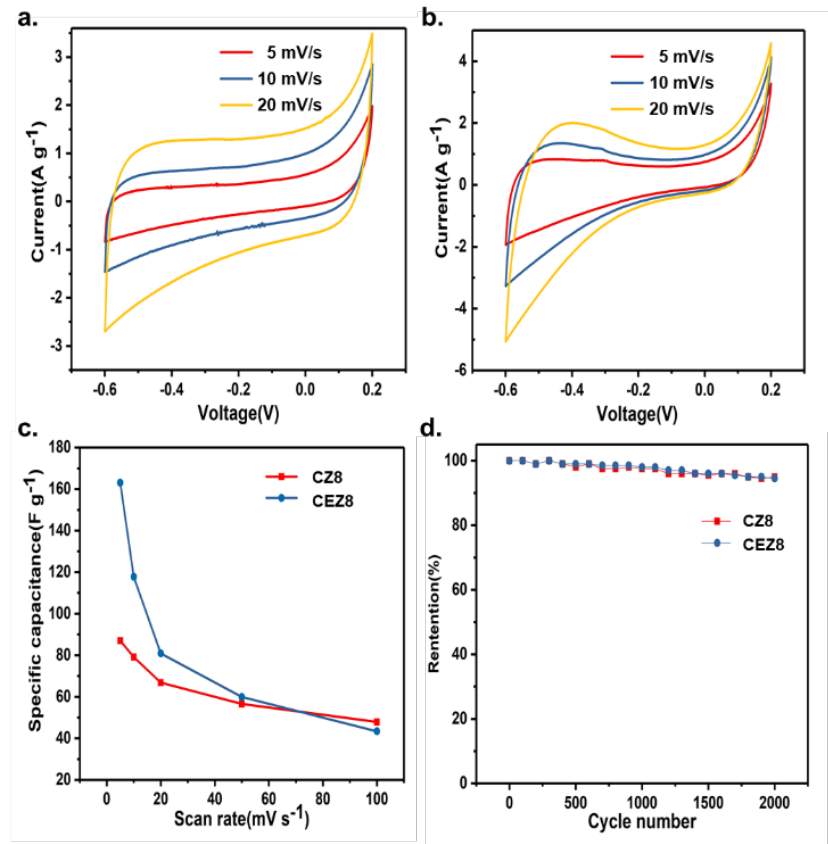

Figure 4. Cyclic voltammograms at different sweep rates of a) CZ8 and b) CEZ8; c) specific capacitance as a function of scan rate for CZ8 and CEZ8; d) cycling stability of CZ8 and CEZ8.

\section{Conclusions}

In this work, we demonstrated a new strategy to prepare hierarchical porous carbons via a straightforward and convenient biomimetic mineralization of ZIF-8 using non-pathogenic and non-toxic bacterial cells. We found that bacterial cell surfaces efficiently nucleate the rapid crystallization of ZIF-8, which produces a crystalline shell that can be carbonized into a material possessing a hierarchy of porosities. The bacterial template is thermally ablated during carbonization and contributes to a higher level of graphitization of the ZIF-8 shell during pyrolysis. The as-prepared CEZ8 possesses superior capacitive performance than carbonized pristine ZIF-8 owing to its high specific surface area, excellent conductivity, and welldeveloped hierarchical porosity. We believe that the size and morphological diversity of bacteria and their high reproduction rate means they hold great promise in templating and fabricating hierarchical porous carbon materials. 


\section{ASSOCIATED CONTENT}

\section{Supporting Information}

Experimental procedure, characterization techniques and additional characterization results are available in supplemented file.

\section{AUTHOR INFORMATION}

\section{Corresponding Author}

Jeremiah. J. Gassensmith

Email: gassensmith@utdallas.edu

Phone: (972) 883-3596

Group Page: www.gassensmithlab.com

\section{Present Addresses}

The University of Texas at Dallas Dept. of Chemistry \& Biochemistry 800 West Campbell Road Mailstop: BS13 Richardson, TX 75080.

\section{Author Contributions}

The manuscript was written through contributions of all authors. All authors have given approval to the final version of the manuscript.

\section{Funding}

J.J.G wishes to thank the Robert A. Welch Foundation [AT-198920190330] for funding this research and the National Science Foundation [CAREER DMR-1654405] for funding the integration of his entire scholarly and educational activities.

\section{REFERENCES}

1. Lee, J.; Kim, J.; Hyeon, T. Recent Progress in the Synthesis of Porous Carbon Materials. Adv. Mater. 2006, 18, 2073-2094.

2. Hu, Y. S.; Adelhelm, P.; Smarsly, B. M.; Hore, S.; Antonietti, M.; Maier, J. Synthesis of Hierarchically Porous Carbon Monoliths with Highly Ordered Microstructure and Their Application in Rechargeable Lithium Batteries with High-Rate Capability. Adv. Funct. Mater. 2007, 17, 1873-1878.

3. Roberts, A. D.; Li, X.; Zhang, H. Porous carbon spheres and monoliths: morphology control, pore size tuning and their applications as Li-ion battery anode materials. Chem. Soc. Rev. 2014, 43, 4341-4356.

4. Zhai, Y.; Dou, Y.; Zhao, D.; Fulvio, P. F.; Mayes, R. T.; Dai, S. Carbon Materials for Chemical Capacitive Energy Storage. Adv. Mater. 2011, 23, 4828-4850.

5. Zhu, C.; Li, H.; Fu, S.; Du, D.; Lin, Y. Highly efficient nonprecious metal catalysts towards oxygen reduction reaction based on three-dimensional porous carbon nanostructures. Chem. Soc. Rev. 2016, 45, 517-531.

6. Wang, C.; Kaneti, Y. V.; Bando, Y.; Lin, J.; Liu, C.; Li, J.; Yamauchi, Y. Metal-organic framework-derived one-dimensional porous or hollow carbon-based nanofibers for energy storage and conversion. Mater. Horiz. 2018, 5, 394-407.

7. Wang, Y.; Wang, Z.; Yu, X.; Li, B.; Kang, F.; He, Y.-B. Hierarchically structured carbon nanomaterials for electrochemical energy storage applications. J. Mater. Res. 2018, 33, 1058-1073.

8. Liang, C.; Li, Z.; Dai, S. Mesoporous Carbon Materials: Synthesis and Modification. Angew. Chem., Int. Ed. 2008, 47, 3696-3717.

9. Furukawa, H.; Cordova, K. E.; O'Keeffe, M.; Yaghi, O. M. The Chemistry and Applications of Metal-Organic Frameworks. Science 2013, 341, 1230444.

10. Yaghi, O. M.; O'Keeffe, M.; Ockwig, N. W.; Chae, H. K.; Eddaoudi, M.; Kim, J. Reticular synthesis and the design of new materials. Nature 2003, 423, 705.

11. Park, K. S.; Ni, Z.; Côté, A. P.; Choi, J. Y.; Huang, R.; UribeRomo, F. J.; Chae, H. K.; O'Keeffe, M.; Yaghi, O. M. Exceptional chemical and thermal stability of zeolitic imidazolate frameworks. Proc. Natl. Acad. Sci. U. S. A. 2006, 103, 10186-10191.
12. Indra, A.; Song, T.; Paik, U. Metal Organic Framework Derived Materials: Progress and Prospects for the Energy Conversion and Storage. Adv. Mater. 2018, 30, 1705146.

13. Zhu, B.; Xia, D.; Zou, R. Metal-organic frameworks and their derivatives as bifunctional electrocatalysts. Coord. Chem. Rev. 2018, 376, $430-448$.

14. Li, X.; Zheng, S.; Jin, L.; Li, Y.; Geng, P.; Xue, H.; Pang, H.; $\mathrm{Xu}, \mathrm{Q}$. Metal-Organic Framework-Derived Carbons for Battery Applications. Adv. Energy Mater. 2018, 8, 1800716.

15. Zhang, H.; Liu, X.; Wu, Y.; Guan, C.; Cheetham, A. K.; Wang, J. MOF-derived nanohybrids for electrocatalysis and energy storage: current status and perspectives. Chem. Commun. 2018, 54, 5268-5288.

16. Xia, W.; Mahmood, A.; Zou, R.; Xu, Q. Metal-organic frameworks and their derived nanostructures for electrochemical energy storage and conversion. Energ Environ Sci. 2015, 8, 1837-1866.

17. Salunkhe, R. R.; Kaneti, Y. V.; Kim, J.; Kim, J. H.; Yamauchi, Y. Nanoarchitectures for Metal-Organic Framework-Derived Nanoporous Carbons toward Supercapacitor Applications. Acc. Chem. Res. 2016, 49, 2796-2806.

18. Wu, X.; Yang, C.; Ge, J. Green synthesis of enzyme/metalorganic framework composites with high stability in protein denaturing solvents. Bioresour. Bioprocess. 2017, 4, 24.

19. Liang, W.; Ricco, R.; Maddigan, N. K.; Dickinson, R. P.; Xu, H.; Li, Q.; Sumby, C. J.; Bell, S. G.; Falcaro, P.; Doonan, C. J. Control of Structure Topology and Spatial Distribution of Biomacromolecules in Protein@ZIF-8 Biocomposites. Chem. Mater. 2018, 30, 1069-1077.

20. Liang, K.; Richardson, J. J.; Doonan, C. J.; Mulet, X.; Ju, Y.; Cui, J.; Caruso, F.; Falcaro, P. An Enzyme-Coated Metal-Organic Framework Shell for Synthetically Adaptive Cell Survival. Angew. Chem., Int. Ed. 2017, 56, 8510-8515.

21. Liang, K.; Richardson, J. J.; Cui, J.; Caruso, F.; Doonan, C. J.; Falcaro, P. Metal-Organic Framework Coatings as Cytoprotective Exoskeletons for Living Cells. Adv. Mater. 2016, 28, 7910-7914.

22. Li, S.; Dharmarwardana, M.; Welch, R. P.; Benjamin, C. E.; Shamir, A. M.; Nielsen, S. O.; Gassensmith, J. J. Investigation of Controlled Growth of Metal-Organic Frameworks on Anisotropic Virus Particles. ACS Appl. Mater. Interfaces 2018, 10, 18161-18169.

23. Li, S.; Dharmarwardana, M.; Welch, R. P.; Ren, Y.; Thompson, C. M.; Smaldone, R. A.; Gassensmith, J. J. Template-Directed Synthesis of Porous and Protective Core-Shell Bionanoparticles. Angew. Chem., Int. Ed. 2016, 55, 10691-10696.

24. Riccò, R.; Liang, W.; Li, S.; Gassensmith, J. J.; Caruso, F.; Doonan, C.; Falcaro, P. Metal-Organic Frameworks for Cell and Virus Biology: A Perspective. ACS Nano 2018, 12, 13-23.

25. Lyu, F.; Zhang, Y.; Zare, R. N.; Ge, J.; Liu, Z. One-Pot Synthesis of Protein-Embedded Metal-Organic Frameworks with Enhanced Biological Activities. Nano Lett. 2014, 14, 5761-5765.

26. Liang, K.; Ricco, R.; Doherty, C. M.; Styles, M. J.; Bell, S.; Kirby, N.; Mudie, S.; Haylock, D.; Hill, A. J.; Doonan, C. J.; Falcaro, P. Biomimetic mineralization of metal-organic frameworks as protective coatings for biomacromolecules. Nat. Commun. 2015, 6, 7240.

27. Li, W.; Zhang, Y.; Xu, Z.; Meng, Q.; Fan, Z.; Ye, S.; Zhang, G. Assembly of MOF Microcapsules with Size-Selective Permeability on Cell Walls. Angew. Chem., Int. Ed. 2015, 55, 955-959.

28. Luzuriaga, M. A.; Welch, R. P.; Dharmarwardana, M.; Benjamin, C. E.; Li, S.; Shahrivarkevishahi, A.; Popal, S.; Tuong, L. H.; Creswell, C. T.; Gassensmith, J. J. Enhanced Stability and Controlled Delivery of MOF-Encapsulated Vaccines and Their Immunogenic Response In Vivo. ACS Appl. Mater. Interfaces 2019, 11, 9740-9746.

29. Zhu, H.; Yin, J.; Wang, X.; Wang, H.; Yang, X. Microorganism-Derived Heteroatom-Doped Carbon Materials for Oxygen Reduction and Supercapacitors. Adv. Funct. Mater. 2013, 23, 1305-1312. 
SYNOPSIS TOC (Word Style "SN_Synopsis_TOC"). If you are submitting your paper to a journal that requires a synopsis graphic and/or synopsis paragraph, see the Instructions for Authors on the journal's homepage for a description of what needs to be provided and for the size requirements of the artwork.

To format double-column figures, schemes, charts, and tables, use the following instructions:

Place the insertion point where you want to change the number of columns

From the Insert menu, choose Break

Under Sections, choose Continuous

Make sure the insertion point is in the new section. From the Format menu, choose Columns

In the Number of Columns box, type 1

Choose the OK button

Now your page is set up so that figures, schemes, charts, and tables can span two columns. These must appear at the top of the page. Be sure to add another section break after the table and change it back to two columns with a spacing of 0.33 in.

Table 1. Example of a Double-Column Table

\begin{tabular}{|l|l|l|l|l|l|l|l|}
\hline Column 1 & Column 2 & Column 3 & Column 4 & Column 5 & Column 6 & Column 7 & Column 8 \\
\hline & & & & & & & \\
& & & & & & & \\
\hline
\end{tabular}

Authors are required to submit a graphic entry for the Table of Contents (TOC) that, in conjunction with the manuscript title, should give the reader a representative idea of one of the following: A key structure, reaction, equation, concept, or theorem, etc., that is discussed in the manuscript. Consult the journal's Instructions for Authors for TOC graphic specifications. 